\title{
Gerencia profesional y contratación pública estratégica: una perspectiva comparada
}

\section{Professional management and strategic public procurement. A comparative perspective}

\author{
Juan José Rastrollo Suárez \\ Universidad de Salamanca (España) \\ ORCID: https://orcid.org/0000-0003-0521-1045 \\ rastrollo@usal.es
}

\begin{abstract}
NOTA BIOGRÁFICA
Profesor Contratado Doctor acreditado Titular de Universidad de Derecho administrativo en la Universidad de Salamanca. Doctor Europeus (Universidad de Salamanca/Universidad de Coimbra) en Derecho y Premio extraordinario de Grado y Doctorado. Secretario de la Revista Documentación Administrativa, del Instituto Nacional de Administración Pública de España. Ha sido profesor invitado, entre otras, en las Universidades de los Andes y Nariño (Colombia), Andina Simón Bolivar (Ecuador) o James Madison de Virginia (Estados Unidos). Consultor y docente para servidores públicos en distintas instituciones de América Latina (Fundación Salvadoreña para el Desarrollo Económico y Social de El Salvador, Contraloría de Costa Rica, Procuraduría de Paraguay). Miembro de los grupos de investigación State and Nation Making in Latin America and Spain (Universidad de Princeton) y Next Generation EU (Universidad de Salamanca) e investigador de la Unidad de Investigación Consolidada de la Junta de Castilla y León (núm. 302). Autor de tres monografías, más de 25 artículos y más de 15 capítulos de libro en revistas y obras colectivas de Europa y América. Sus principales líneas de investigación son el Derecho urbanístico, la contratación pública y el empleo público y en general la reforma y modernización de la Administración pública en Europa y América Latina.
\end{abstract}

\section{RESUMEN}

A partir del examen de distintos informes, identificamos las dificultades para la profesionalización de la contratación pública. En base a ello, revisamos experiencias de éxito desarrolladas por algunos de los mejores sistemas de compra pública del mundo, identificando buenas prácticas a tener en cuenta en el desarrollo futuro de la estrategia nacional de contratación pública, en conexión con el contenido de la Recomendación de la Unión Europea sobre profesionalización de la contratación pública de 2017.

\section{PALABRAS CLAVE}

Profesionalización; contratación pública estratégica; empleo público; innovación; Unión Europea.

\begin{abstract}
Taking as our point of departure the examination of diverse reports, we identify difficulties for the professionalization of public procurement. On this basis, we revise successful experiences developed by some of the best practices in procurement systems around the world, identifying best practices to take into account for the future development of a national strategy of public procurement. We connect this revision with the contents of the Recommendation of the European Union of 2017 on professionalization of public procurement.
\end{abstract}

\section{KEYWORDS}

Professionalization; strategic public procurement; public employment; innovation; European Union. 


\begin{abstract}
SUMARIO
I. INTRODUCCIÓN: CONTRATACIÓN PÚBLICA ESTRATÉGICA Y EMPLEO PÚBLICO. II. DEBILIDADES DE LA CONTRATACIÓN PÚBLICA. III. PROCESOS DE PROFESIONALIZACIÓN DE LA CONTRATACIÓN PÚBLICA EN PERSPECTIVA COMPARADA. 1. LA GERENCIA PROFESIONAL DE LA CONTRATACIÓN PÚBLICA. 2. LA RECOMENDACIÓN 2017/1805 «CONSTRUIR UNA ARQUITECTURA PARA LA PROFESIONALIZACIÓN DE LA CONTRATACIÓN PÚBLICA». 2.1. Definición de una política para la profesionalización de la contratación pública. 2.2. La mejora de la formación y la gestión profesional en el ámbito de los recursos humanos. 2.3. El suministro de herramientas y metodologías adecuadas. III. CONCLUSIONES. REFERENCIAS BIBLIOGRÁFICAS.
\end{abstract}

\title{
I. INTRODUCCIÓN: CONTRATACIÓN PÚBLICA ESTRATÉGICA Y EMPLEO PÚBLICO
}

El nivel de profesionalización de la función pública de un país afecta directamente a su desarrollo económico y social (Evans y Rauch, 1999). El índice de efectividad internacional del servicio civil (InCiSE) de 2019 promovido por la Blavatnik School of Government de la Universidad de Oxford sitúa a España ligeramente por encima de la media de los treinta y ocho países analizados. Sin embargo, se encuentra por debajo de la media en relación con la gestión de recursos humanos o de la contratación pública ${ }^{1}$. Este último es uno de los ámbitos de actividad administrativa en los que resulta más importante garantizar una gestión profesional, especialmente en el entorno de la contratación pública europea, que considera este un elemento clave para prevenir la corrupción (Malaret i García, 2016, p. 45).

La contratación pública se caracteriza por una gran complejidad técnica, el reconocimiento de grandes márgenes de discrecionalidad y la vinculación a elevadas partidas presupuestarias, siendo por ello especialmente sensible a prácticas corruptas (Wayan Rustiarini, et al., 2019). El riesgo disminuye significativamente cuando quienes la impulsan y supervisan son seleccionados en base a criterios vinculados al mérito y la capacidad, son adecuadamente formados al inicio y durante su carrera y cuentan con la autonomía suficiente para tomar sus decisiones de forma independiente (Charrón et al., 2017). Existe por tanto una relación directa entre la capacidad e independencia de quienes toman las decisiones y la preservación del principio de integridad en la contratación pública (Bandiera et al., 2009 o Cerrillo i Martínez, 2014, p. 165)².

La aparición del concepto de "compra pública estratégica» a raíz de la aprobación de las Directivas sobre la materia de 2014 y su incorporación a la Ley 9/2017, de 8 de noviembre, de Contratos del Sector Público (en adelante LCSP) en directa conexión con la estrategia Europa 2020, ha hecho de esta tarea algo aún más complejo (Gimeno Feliú, 2016, pp. 15-127) ${ }^{3}$. Los nuevos procedimientos y exigencias para participar en las licitaciones y el incremento y la variedad de criterios de adjudicación, inciden directamente en los empleados públicos implicados en los procedimientos ${ }^{4}$. En este contexto, el artículo 334.1.e) LCSP reconoció como uno de los aspectos en los que debía incidir la Estrategia Nacional de Contratación pública (en adelante ENCP) -cuyo desarrollo es competencia de la Oficina Independiente de Regulación y Supervisión de la Contratación (en adelante OIRESCON)- la promoción de la «profesionalización en contratación pública mediante actuaciones de formación del personal del sector público, especialmente en aquellas entidades que carecen de personal especializado, mediante el diseño de un plan específico en materia de formación en contratación pública y otras actuaciones complementarias de promoción de la profesionalización en la contratación pública». Además, la existencia de problemas globales que requieren de actuaciones inmediatas puesta de manifiesto durante la crisis sanitaria ha supuesto un punto de inflexión. Las situaciones de emergencia en las que es preciso realizar compras en un mercado internacional en el que múltiples demandantes pugnan por recursos escasos haciendo uso de procedimientos sometidos a priori a menos controles, han

\footnotetext{
1 Dentro de la función pública española destacan por su nivel de profesionalización según el índice quienes se dedican a la recaudación de impuestos, además de su capacidad de reacción frente a crisis, la gestión del riesgo, el uso de nuevas tecnologías o lo relativo a la gestión fiscal y financiera (Blavatnik School of Government, 2020, p. 62).

2 Así se lleva reclamando desde instancias internacionales desde la primera década del siglo XXI, véase en tal sentido (OCDE, 2009b, p. 61).

3 Explicitada en la Comunicación de la Comisión "Europa 2020. Una estrategia para un crecimiento inteligente, sostenible e integrador" de 3 de marzo de 2010, COM (2010) 2020 final.

4 En este contexto los considerandos 59 y 69 de la Directiva 2014/24/UE del Parlamento Europeo y del Consejo, de 26 de febrero de 2014, sobre contratación pública y por la que se deroga la Directiva 2004/18/CE (en adelante Directiva 2014/24) ligan a la agregación de demanda por parte de los compradores públicos y las técnicas de recentralización de compras a la profesionalización.
} 
puesto en evidencia que contar con profesionales suficientemente capaces y formados resulta decisivo para afrontar los riesgos imprevistos a los que se enfrenta la sociedad.

A lo largo de este trabajo examinamos las debilidades que encontramos en el sistema de contratación pública, identificando los aspectos en los que cabría mejorar para lograr una profesionalización conectada con la compra pública estratégica (Steinfeld et al., 2015, p. 523). La Recomendación 2017/1805 de Comisión Europea de 3 de octubre de 2017 «Construir una arquitectura para la profesionalización de la contratación pública» (en adelante Recomendación) exige elaborar estrategias de profesionalización y confrontarlas con las de otros Estados miembros y las que se dan en el contexto internacional por lo que, con base en las debilidades localizadas, revisamos experiencias de éxito desarrolladas en el marco de algunos de los mejores sistemas de compra pública del mundo, identificando distintas prácticas a tener en cuenta de cara al desarrollo futuro de la ENCP.

\section{DEBILIDADES DE LA CONTRATACIÓN PÚBLICA}

En 2016 la Comisión Europea hizo público el informe basado en entrevistas a empleados públicos Stock-taking of administrative capacity, systems and practices across the EU to ensure the compliance and quality of public procurement involving European Structural and Investment (ESI) Funds (Balance de la capacidad, los sistemas y las practicas administrativas en la UE para garantizar la conformidad y la calidad de la contratación pública que implique la participación de los Fondos Estructurales y de Inversión Europeos). Uno de sus apartados se consagró al análisis de las dificultades a las que se enfrenta la contratación en los países de la UE, extrayéndose como principales conclusiones que:

- Aunque la mayor parte de los empleados cuentan con formación en Derecho o Economía, tienen mucha menor capacitación en áreas como las de Gestión Administrativa o Ciencia de la Administración, lo que podría resultar clave para impulsar la contratación pública estratégica. Cuentan, además, con una nula o muy escasa experiencia en el sector privado, lo que puede limitar su capacidad para entender el funcionamiento de los mercados de contratación pública desde una perspectiva integral (CE, 2016, p. 37). Además, la rotación entre puestos de trabajo en el sector es muy escasa (España es el sexto país de los analizados en los que en menor medida se producía) (CE, 2016, p. 38).

- Si bien la formación era relativamente habitual (solo una cuarta parte de los gerentes declaró que los empleados a su cargo no habían asistido a cursos o conferencias en el último año), la capacitación se orientaba fundamentalmente a cuestiones de carácter general y tan solo el $10 \%$ se había centrado en la lucha contra la corrupción. España es el país de la Unión en que menos empleados se habían beneficiado de cursos de formación especializados (CE, 2016, p. 39).

- Es habitual el uso de sistemas, manuales y otras herramientas en los procedimientos (en España menos del cinco por ciento de los empleados están excluidos del acceso a las mismas) (CE, 2016, pp. 41-43). Sin embargo, las consultas online, las plataformas electrónicas y otro tipo de páginas tan solo están disponibles en los especialmente complejos (CE, 2016, p. 47).

- La notificación es la práctica con mayor implantación dentro de la administración electrónica (realizada por tres cuartos de los encuestados). Alrededor de un $70 \%$ facilita el acceso documental por vía electrónica, mientras la presentación de ofertas se da tan solo en alrededor de un $45 \%$ de los casos (en España el porcentaje supera por poco el $35 \%$ ).

- Sólo es habitual la recopilación de la información más básica (presupuesto de contrato y tipo de procedimiento utilizado). Otros aspectos que podrían resultar de interés no se recogen ni almacenan habitualmente. Una cuarta parte no monitoreaba ningún dato relativo al contrato y poco más de la mitad publicaban los datos recopilados, si bien España es uno de los países en los que se hacen públicos los datos de manera más habitual, con cerca de un $70 \%$ (CE, 2016, p. 51). Ello resulta especialmente preocupante teniendo en cuenta la incidencia directa que puede tener la publicidad en la contratación, pudiendo reducir los costes en las adquisiciones hasta en un 0,7\% y facilitar la selección entre un mayor número de licitadores (Coviello y Mariniello, 2014, p. 86).

- La formación del personal es el instrumento más utilizado para la prevención de la corrupción (característico de más del $40 \%$ de las organizaciones) siendo otros como el consejo legal, la adopción de estrategias de integridad o la recopilación y monitoreo de datos específico mucho menos habituales. 
- El principal problema señalado por los gestores es la complejidad de normas y reglamentos, seguida del exceso en la carga de trabajo, la limitación de tiempo y la abundancia de cambios legislativos, lo cual es especialmente acusado en las Administraciones que carecían de personal formado (CE, 2016, pp. 53-54).

Por su parte, el Informe anual de supervisión de la contratación pública en España de 2019 de la OIRESCON, señaló la necesidad de hacer del incremento de la profesionalización y de la integración de personal cualificado en aquellas entidades que carecen de él -fundamentalmente las entidades locales- uno de los objetivos centrales de la ENCP. Como indicó, el contexto del empleo público en España requiere que la profesionalización se proyecte a lo largo de toda la carrera administrativa, vinculándose a la existencia de incentivos (OIRESCON, 2020, p. 194).

En cuanto a la capacitación, destacó la necesidad de garantizar una oferta formativa adecuada, que se reveló insuficiente en el momento de promulgarse la LCSP. Además, señaló la necesidad de profundizar en la especialización -clave en el entorno de la contratación pública estratégica-, por lo que para su diseño resulta fundamental la colaboración con las distintas Administraciones (estatal, autonómica, local e institucional). Indicó que la formación debe someterse a criterios de calidad, que permitan su mejora continua, teniendo en cuenta las concretas necesidades que en cada momento demanden los profesionales. El informe puso de relevancia la conveniencia de invertir en este ámbito por el beneficio económico que supone a medio plazo, habida cuenta de la directa relación entre profesionalización e incremento de eficacia en la contratación (OIRESCON, 2010, p. 195).

\section{PROCESOS DE PROFESIONALIZACIÓN DE LA CONTRATACIÓN PÚBLICA EN PERSPECTIVA COMPARADA}

\section{La gerencia profesional en la contratación pública}

En 2009, la OCDE planteó entre los objetivos de cara a profesionalizar la contratación -que identificaba como base necesaria para garantizar la integridad en las compras públicas-, la capacitación de los empleados para adaptarse a un entorno cambiante, sustituyendo el concepto de funcionario encargado de contrataciones por el de gerente de contratos (OCDE, 2009b). Ello implica de una parte atraer talento, de otra dotar a los seleccionados de una formación adecuada para dar respuesta a la creciente complejidad a la que se enfrentan y, por último, proporcionarles la capacitación precisa para poder responder a los retos a los que se enfrentan a partir la centralización de la información y la monitorización de los procesos de contratación.

No obstante, quienes se dedican a la contratación pública en España forman un conjunto heterogéneo de empleados (Cantero Martínez, 2020, p. 224). En él se incluyen a quienes forman parte de los órganos de contratación de elección política, pero también a sus asistentes, que cuentan con un perfil mayoritariamente técnico (como los integrantes de las mesas y juntas de contratación) (artículos 323 y ss. LCSP), al responsable del contrato (artículo 62 LCSP), a los miembros de la OIRESCON (artículo 332 LCSP) o a quienes se dedican a la fiscalización de la contratación pública, incluyendo a jueces y magistrados. Cada uno de ellos cuenta con funciones y regímenes laborales diferenciados. En su mayoría, combinan la participación en procedimientos de contratación o su fiscalización con otro tipo de actividades al servicio de la Administración, a veces de muy variado tipo, situación que comparten otros países de la Unión Europea, como Alemania (OCDE, 2019a).

En otros países del mundo, sin embargo, se ha asimilado la contratación pública a una profesión especializada. La identificación como «profesional» de la contratación implica, a su vez, el reconocimiento de un salario acorde con la responsabilidad que se lleva a cabo, la adquisición de cierto estatus social o de que se trabaja para el interés general (Krause, 1996). Ello está directamente ligado a la existencia de requerimientos de especialización, sistemas de selección competitivos y garantía de independencia en la toma de decisiones (Kline, 1981; Christensen, 1994 y Steinfeld et al., 2016). No obstante, no todos los modelos de especialización resultan fácilmente trasladables a la idiosincrasia propia de la contratación pública española como el estadounidense, donde los purchasing managers, buyers, and purchasing agents se consideran por la US Bureau of Labor Statistics como una única profesión, sin diferenciar entre los que desarrollan esta actividad en el sector público o privado (Steinfeld et al., 2015, p. 510).

Un posible modelo a seguir podría ser el constituido en el Reino Unido por la Government Commercial Function (GCF), una sección del Civil Service que forma parte de la Oficina del Gabinete del Gobierno 
conformada por un cuerpo de especialistas de alrededor de 40.000 empleados públicos, cuya función es apoyar a distintos Departamentos gubernamentales y al Crown Commercial Service respecto a la gestión y planificación de las compras públicas 5 . Está formado por especialistas en contratación que, a su vez, se encuentran respaldados por otros empleados públicos especializados en diversas áreas.

Bajo el mando del Government Chief Commercial Officer enfocan su actividad en auxiliar a la Administración respecto a procedimientos complejos, licitaciones internacionales, recopilación de información, controles o elaboración de buenas prácticas a lo largo de todo el procedimiento de adquisición: desde la planificación a la gestión. A través de esta red de empleados se impulsa, además, el programa Commercial Capability, orientado a desarrollar la capacidad en materia de contratación pública del resto de empleados de la Administración. La GCF cuenta con una guía de estándares requeridos para formar parte de las distintas categorías de su cuerpo de empleados (Commercial Lead, Associate Commercial Specialist, Commercial Specialist o Senior Commercial Specialist). Los de rango superior (más de séptimo grado) pasan a depender de la Government Commercial Organisation (GCO), interna a la GCF. Esta última está formada por alrededor de 250 empleados, siendo su principal misión apoyar a la Administración gubernamental en los procedimientos más complejos.

El de gerente de contratos se configuraría así como un cuerpo de empleados públicos especializado en la asistencia a las distintas Administraciones para la puesta en marcha y desarrollo de los procedimientos de licitación. En su diseño habría que tener en cuenta las especialidades de la contratación y, en particular, el nivel de exposición a la corrupción al que se enfrentaría: la transparencia en el proceso de selección está directamente vinculada a la independencia con la que los profesionales cuenten a la hora de tomar sus decisiones, de lo que dependerá en buena medida la eficacia del sistema de contratación (Cooper 2008: 271).

\section{La Recomendación $2017 / 1805$ «Construir una arquitectura para la profesionalización de la contratación pública»}

La Resolución del Parlamento Europeo de 25 de octubre de 2011 sobre la modernización de la contratación pública, puso de manifiesto que una mejor formación de adjudicatarios y licitadores incrementaría la competencia este mercado (en la que solo un $1,4 \%$ de los contratos eran adjudicados a empresas de otros Estados) (Sanmartín Mora, 2012). Seis años después la Recomendación pasó a definir la profesionalización como la actividad tendente a conseguir una «mejora general de toda la gama de cualificaciones y competencias profesionales, conocimientos y experiencia de las personas que realizan o participan en tareas relacionadas con la contratación». A su vez, el Dictamen del Comité Económico y Social Europeo respecto a la misma, re refiere a ella como la necesidad de garantizar que los empleados «posean las competencias profesionales y los conocimientos técnicos y procedimentales necesarios para cumplir con la normativa y asegurar una elevada calidad en términos de innovación y sostenibilidad y la mejor relación calidad-precio posible de la inversión, también desde el punto de vista de la responsabilidad social» (Comité Económico y Social Europeo, 2018, p. 48).

La Recomendación diseña una estrategia para la profesionalización centrada en objetivos más cualitativos que cuantitativos y dirigida a todos los empleados públicos implicados en la contratación (Cantero Martínez, 2020, p. 208). Además, como se deduce a partir de la definición empleada, es concebida con un carácter transversal y heterogéneo, requiriendo herramientas focalizadas en diferentes aspectos. La estrategia se divide en tres ejes fundamentales: el desarrollo de una arquitectura política adecuada, la formación y gestión de la carrera profesional y el establecimiento de sistemas compuestos por herramientas y metodologías de apoyo a la gestión profesional de la contratación pública.

\subsection{Definición de una política para la profesionalización de la contratación pública}

Supone la necesidad de incorporar la profesionalización de la contratación pública a la agenda política, haciendo de la misma un proyecto que aúne el consenso suficiente para ser desarrollado a medio y largo plazo (Cantero Martínez, 2020, p. 208). Dentro de este eje, la Recomendación hace referencia a dos aspectos:

En primer lugar, a la necesidad de elaborar estrategias de profesionalización a largo plazo adaptadas a las «necesidades, recursos y estructuras administrativas» concretos con el fin de «atraer, desarrollar y rete-

\footnotetext{
5 Government Commercial Function. https://www.gov.uk/government/organisations/government-commercial-function
} 
ner competencias, centrarse en el rendimiento y los resultados estratégicos y aprovechar las herramientas y técnicas disponibles». Se advierte la necesidad de proyectar las técnicas sobre el conjunto de Administraciones, coordinarlas con el resto de políticas del sector público y confrontarlas con las de otros Estados miembros y las que se dan en el contexto internacional. En segundo lugar, a la necesidad de que animar y apoyar a las administraciones en su implementación, fomentando la cooperación entre servicios y entidades o autoridades de contratación y aprovechando la experiencia de instituciones de formación, centrales de compra y organizaciones profesionales.

Impulsar una estrategia de profesionalización requiere ponerla en marcha a partir de instrumentos que permitan atraer talento, al margen de los tradicionales sistemas de selección de empleados públicos en España y los sacrificios económicos, personales y en cuanto a tiempo que conllevan (Jiménez Asensio, 2018, p. 10). En este sentido, existen experiencias innovadoras que podrían tenerse en cuenta al diseñar un modelo de selección de gerentes de la contratación (Rivero Ortega, 2012, p. 87; Mapelli Marchena, 2018). Si bien estos instrumentos alternativos a la oposición -que ha venido a garantizar en buena medida la igualdad en el acceso al empleo público en España durante más de un siglo, mérito nada desdeñable- deben ser adaptados a las particulares circunstancias económicas, institucionales, jurídicas y sociales. Cualquier paso en falso en este sentido podría poner en riesgo el necesario respeto a los principios de igualdad material, mérito y capacidad en la selección (Rastrollo Suárez, 2018, p. 240).

El empleo público de Canadá es el mejor valorado por el ranking InCISE 2019 en lo relativo a la gestión de recursos humanos y ocupa el segundo lugar de los países analizados en lo relativo a la integridad de los empleados, de tal manera que el empleo público es una de las oportunidades de desarrollo profesional más demandadas por los estudiantes universitarios del país (Eddy y Gosset, p. 2013). La función pública canadiense vivió un punto de inflexión en el año 2003 con la aprobación de la Public Service Modernization Act. La norma tuvo como punto de partida el Report of the Auditor General of Canada de 2000, que vino a poner de manifiesto la necesidad de reforma en relación a aspectos como la racionalidad, la flexibilidad o la autoridad de los gerentes ${ }^{6}$. Desde entonces, distintas normas han seguido impulsado una continua reforma a partir de distintos informes anuales ${ }^{7}$ y la actuación de la Public Service Commission of Canada, agencia independiente que tiene entre sus principales funciones preservar el mérito como criterio central de selección y progreso en el ámbito del empleo público.

Una herramienta novedosa con las que cuenta el sistema de empleo público canadiense es la Talent Cloud o nube de talento. Se trata de un proyecto piloto basado una plataforma electrónica en la que cualquier aspirante puede registrarse proporcionando su nombre y una dirección de correo electrónico y ser seleccionado para una oferta de trabajo de cualquiera de los Departamentos del gobierno asociados al proyecto, entre los que se encuentra el Public Services and Procurement Canada, encargado de las compras públicas. La plataforma permite que los postulantes se comuniquen directamente con los gerentes respecto a las ofertas de trabajo, manifestando sus capacidades para el desempeño del puesto sin que puedan ser excluidos a priori del proceso de selección por no contar con un currículum convencional respecto al empleo solicitado.

Las postulaciones forman parte de una base de datos a disposición de los Departamentos adheridos y en la medida en que resulten interesantes a los gerentes, los aspirantes pueden ser contactados para realizar exámenes o entrevistas. Con independencia de conseguir o no el trabajo, el gerente con el que el aspirante entra en contacto puede certificar, en la medida en que supere las pruebas y la entrevista, la capacidad de este último para ocupar un puesto de trabajo del tipo del que se oferta de cara a futuras ofertas.

También es destacable el ejemplo belga. El Bureau de sélection de l'administration fédérale u Oficina Federal de Selección y reclutamiento (SELOR) dependiente del Service public fédéral personnel et organisation es la encargada de llevar a cabo los procesos de selección ${ }^{8}$. Las ofertas -cuya información detallada puede recibirse periódicamente a través de una lista de correo- se publican en su página electrónica, con referencia a la titulación y la experiencia requeridas. Para la postulación tan solo es preciso registrarse en la plataforma, crear una cuenta y cargar los certificados requeridos. Tras verificar el cumplimiento de los requisitos, se produce el registro para la realización de los exámenes.

El examen genérico no se exige a quienes ya son funcionarios, miembros del poder judicial o pertenecen al ejército (interne markt). Se realiza en las dependencias de SELOR durante un máximo de tres horas

6 Junto a otros informes, como los del Advisory Committee on Senior Level Retention and Compensation de 2000 o del Advisory Committee on Labour-Management Relations in the Federal Public Service «Working Together in the Public Interest» de 2001.

7 Informes del Privy Councl Office of Canada de 2008 y 2009.

8 Selor. Bureau de Sélection de l'Administration Fédérale. https://www.selor.be/nl/ 
y media y consiste en tres pruebas: Postbakoefening (o ejercicio de simulación de bandeja de correo, que mide la capacidad de discriminación entre distintas tareas en función de su importancia), Situationele beoordelingstest (o prueba de evaluación situacional, que mide las reacciones ante distintas situaciones) y Abstract redeneervermogen (destinado a evaluar la capacidad de razonamiento abstracto, al medir la capacidad para derivar reglas generales de la información abstracta recibida al aplicarla a un determinado supuesto). El resultado se comunica mediante la plataforma después de una semana, junto a un «informe de retroalimentación». Si se supera, no será preciso volver a realizarlo durante tres años. De lo contrario, el aspirante puede realizarlo de nuevo transcurridos seis meses.

En el específico las pruebas están vinculadas a la descripción del puesto y consisten generalmente en un test de conocimientos y una entrevista. Son realizadas por la institución a la que se adscribe la vacante. El resultado, junto a la retroalimentación, se comunica en un plazo de dos meses. SELOR elabora una lista de quienes han superado las pruebas clasificándolos en función de su puntuación, que permanece vigente para la institución que comunicó la vacante durante un periodo de tiempo preestablecido. A través de la plataforma, el candidato puede recibir ofertas que aceptará o no sin perder su posición en la lista que se abrirá a otras instituciones con vacantes de perfil similar transcurridos tres meses. En este caso se precisará una prueba adicional, generalmente consistente en la realización de una entrevista. Los aspirantes a funcionarios de carrera precisan superar un periodo de prueba de un año, no exigido a los contratados laborales.

En Irlanda, el Public Appointments Service (o PAS) es la agencia central de reclutamiento regida por las Public Service Management (Recruitment and Appointments) Acts de 2004 a 2013'. Da asistencia a las distintas Administraciones y organismos públicos a la hora de llevar a cabo procesos de selección y las coordina en base a lo establecido en los Códigos de Prácticas y las directrices emanadas de la Commision for Public Service Appointments. El proceso comienza con la convocatoria publicada en la página electrónica de la agencia. Generalmente se rellena un formulario en línea que contiene una lista de preguntas diseñadas para identificar a los candidatos idóneos (solicitando información, por ejemplo, sobre los anteriores puestos desempeñados). Continúa habitualmente con un test en línea supervisado (vigilado mediante un mecanismo remoto). Las áreas en las que se examina a los aspirantes varían en función del tipo de puesto, dividiéndose habitualmente entre las de razonamiento lingüístico (para medir la capacidad para entender textos y el analizar argumentos), numérico (para medir la capacidad para analizar y tomar decisiones a partir de datos matemáticos) y simulación de trabajo (para medir la capacidad para enfrentarse a situaciones laborales concretas). El proceso concluye con una entrevista.

El Reino Unido cuenta con un programa para captar a futuros empleados públicos de entre los expedientes más destacados de la Universidad, cuando los estudiantes aún no han concluido sus estudios (García Jiménez, 2017, p. 295). A través del programa Fast Stream se preselecciona a los que cuentan con mayor calificación durante los dos últimos años de carrera. La solicitud se envía por vía electrónica tras registrarse y rellenar una serie de cuestionarios. Posteriormente, los aspirantes realizan una entrevista telefónica. Después, los seleccionados deben elegir cuatro de entre los quince perfiles que componen el programa (ofreciendo algunos de ellos una formación complementaria) y llevar a cabo una serie de prácticas retribuidas en los meses de verano, lo que posibilita el conocimiento directo de las actividades a realizar en los distintos puestos en los que el postulante va rotando dentro de distintos Departamentos. Al concluir las prácticas y siempre que hayan superado esta fase acceden al Fast Pass (vía preferente para llegar a la fase de selección) pudiendo realizar un curso de preparación avanzado para superar el Civil Service Fast Stream). Existen, además, subprogramas específicos dirigidos a personas con diversidad funcional (Early Diversity Internship Programme) o provenientes de familias con un bajo índice de renta (Summer Diversity Internship Programme).

Una vez seleccionados, dada la complejidad y relevancia de la actividad, resulta fundamental proporcionar a los empleados una capacitación inicial y específica que en el marco de la contratación pública estratégica debe vincularse a muy distintos aspectos, que van más allá de los meramente técnicos.

Por ejemplo, en Australia, el Procurement Certification Program (PCP) otorga una certificación otorgada por el Institute of Public Administration (IPAA) ${ }^{10}$. Este se divide en cuatro niveles: los dos primeros están diseñados para el personal de la Administración que participa en procedimientos de licitación de importe y riesgo medios (Procurement Fundamentals e Intermediate Procurement) mientras los dos siguientes están

9 Public Appointments Service. https://www.publicjobs.ie/en/foi/326-information-about-the-public-appointments-service

10 QLD Procurement. https://www.nsw.ipaa.org.au/Shared_Content/Events/Event_Display.aspx?WebsiteKey=1ece817c-d23e-4b21a7bd-d835b2dc19f0\&EventKey=NOCODE 
dirigidos a aquellos empleados públicos llamados a participar en procedimientos de contratación pública de una mayor complegidad (Diploma of Government-Procurement and Contracting o Advanced Diploma of Government Procurement and Contracting) ${ }^{11}$. Mientras tanto en Canadá, es preciso superar un plan de estudios de capacitación en contratación pública de carácter básico, continuamente actualizado conforme a las modificaciones en la legislación sobre contratos desde la aprobación de la Directive on the Administration of Required Training de 2017 sustituida en 2020 por la Directive on Mandatory Training (Gobierno de Canadá, s. f.).

Por su parte, obtener la condición de Chief Acquisitons Officer en Estados Unidos requiere superar un programa de capacitación impulsado por el Federal Acquisitions Institute e impartido en colaboración con entidades privadas. Este conduce a la obtención de la Federal Acquisition Certification for Contracting Officer's Representatives (FAC-COR) y se compone de tres niveles: contracting officers, contract officer representatives y program and project managers a superar en un plazo máximo de veinticuatro meses. El Federal Acquisition Institute determina el nivel de los participantes en función de las competencias demostradas. La incorporación al puesto de trabajo se produce a lo largo del siguiente año (Federal Acquisiton Institute, 2019).

\subsection{La mejora de la formación y la gestión profesional en el ámbito de los recursos humanos}

El segundo de los ejes a los que hace referencia la Recomendación gira en torno a la mejora de la formación y la gestión profesional de los recursos humanos intervinientes en los procesos de contratación y hace referencia a tres aspectos:

En primer lugar, a la identificación y definición de las capacidades y competencias en las que deben formarse los profesionales de la contratación, dada la naturaleza multidisciplinar de los proyectos. Ello implica la necesidad de diseñar marcos de capacidades y competencias como base para el diseño de programas de formación, además de un marco común a escala europea. En segundo término, al diseño de programas de formación (inicial y continua) adecuados, teniendo en cuenta la evaluación de datos y necesidades y los marcos de competencias disponibles. Ello supone desarrollar una oferta de formación a distintos niveles e incorporar soluciones y herramientas innovadoras e interactivas para el aprendizaje, aprovechando la cooperación académica y la investigación. En tercer término, al impulso a una buena gestión de los recursos humanos, complementada con programas de motivación específicos y planificación de la carrera profesional. Este es un pilar fundamental para impulsar una mayor y mejor dedicación y requiere del establecimiento de programas de reconocimiento y certificación, estructuras profesionales, incentivos institucionales y apoyo político o premios a la excelencia.

La monitorización del sistema de compras públicas resulta fundamental a la hora de desarrollar este eje. En tal sentido resulta destacable la experiencia llevada a cabo en Escocia a través del Procurement competency framework (en adelante PCF) o marco de competencia nacional de adquisiciones. Este identifica las competencias que deben reunir los empleados del Scottish Procurement and Property Directorate o Departamento de contratación pública, para realizar sus funciones en desarrollo de las licitaciones. Estas se determinan en base a los estándares establecidos por el Chartered Institute of Procurement and Supply, organización profesional que agrupa a los profesionales dedicados a la contratación en el Reino Unido. El PCF está, a su vez, vinculado a una herramienta en línea que permite la autoevaluación (simple o personalizada) de los empleados, identifica las necesidades en materia de formación y permite planificar el desarrollo futuro de la carrera profesional (OCDE, 2019b).

Además, resulta fundamental impulsar la evaluación del desempeño en el ámbito de los empleados dedicados a la contratación cuyo diseño dependerá de una elaboración adecuada de los marcos de capacidades y competencias, teniendo en cuenta, en todo caso, lo singular de la actividad que desarrollan y las particularidades de la organización en la que trabajan. Nos referimos a cuestiones como la necesidad de favorecer la participación ciudadana en la determinación de los objetivos y su consecución, la selección de parámetros de evaluación vinculados al interés general y no contaminados por restricciones presupuestarias y programaciones políticas limitadas en el tiempo y la articulación de un sistema de recursos respetuoso con los derechos de los empleados públicos (Rastrollo Suárez, 2018). Una de sus principales razones de ser es localizar las debilidades y fortalezas de la organización, permitiendo a la Administración aprovechar el potencial de sus

\footnotetext{
11 https://www.myskills.gov.au/courses/details?Code=PSP50616
} 
empleados incrementando la eficacia y la eficiencia en su actuación, así como servir de fundamento para el diseño de programas de formación específica que permitan superar las limitaciones identificadas.

También encontramos modelos de éxito a nivel internacional en relación a la formación continua de los profesionales dedicados a la contratación. La falta de formación específica se supera en algunos países a partir de los programas impartidos por instituciones especializadas. En Estados Unidos destaca el Digital IT acquisiton Professional Training (DITAP), curso inmersivo diseñado por el United Stated Digital Service (USDS) y la Office of Federal Procurement Policy (OFPP). Superarlo conduce a la obtención del la Federal Acquisiton Certification in Contracting Core-Plus Specialization in Digital Services (FAC-C-DS) (TechFAR Hub, 2020). Por su parte en Canadá, el Certification Program for Procurement and Materiel Management Communities in the Government impulsado por el Acquired Services and Assets Sector Communities Management Office, dependiente de la Office of the Comptroller General es un programa no obligatorio para formar en materia de contratación a los empleados públicos canadienses dividido en tres niveles: I para ser especialista en adquisiciones junior, II para ser especialista en adquisiciones senior y III para ser especialista en gestión de materiales (Treasury Board of Canada Secretariat, 2019).

En algunos países existen centros especializados en el desarrollo de competencias en ámbitos específicos de la contratación. Destacan dos instituciones alemanas: el Centro de competencia para las adquisiciones sostenibles (Kompetenzstelle für nachhaltige Beschaffung, en adelante KNB) y el Centro de Competencia para las Adquisiciones Innovadoras (Kompetenzzentrum innovative Beschaffung, en adelante KOINNO) (OCDE, 2019a). Además de formar a especialistas, sirven para aliviar la elevada carga administrativa que para las entidades locales supone alinearse con este modelo de contratación ${ }^{12}$. Los objetivos de contratación pública estratégica fijados en las distintas administraciones varían (mientras en el ámbito estatal se priman objetivos vinculados a la innovación, la sostenibilidad o la transparencia, en los niveles locales es mayor la preocupación por la integración de las pequeñas y medianas empresas en los procesos de contratación) (Glas et al., 2017, p. 572). La existencia de instituciones que sirvan de soporte para el desarrollo de una contratación pública estratégica adaptada a las necesidades y objetivos concretos de cada administración y coordinen su actividad resulta por tanto decisiva.

EI KNB, dependiente de la Oficina de Adquisiciones del Ministerio del Interior, asiste a las distintas administraciones en materia de compra sostenible ${ }^{13}$. Desarrolla su actividad en buena medida a través de la plataforma de información alojada en su página electrónica ofrece soporte a las actividades desarrolladas por el Centro. Entre estas se encuentran informar y formar sobre compras sostenibles, fundamentalmente a través del uso de nuevas tecnologías. Su comité de expertos desarrolla continuamente ideas y enfoques para impulsarla y las distribuye mediante distintos canales con los que cuenta la institución, que la conectan con las diferentes administraciones del país.

EI KOINNO, impulsado por la Asociación alemana de gestión de materiales, compras y logística del Ministerio de economía y energía, tiene como principal finalidad el fomento de la compra pública innovadora. Cuenta con diversas áreas de actuación ${ }^{14}$. En primer término, asesora a las Administraciones en cuestiones jurídicas y económicas relacionadas con la compra innovadora. En este contexto, presta apoyo en relación con el análisis organizativo, la optimización y utilización de procedimientos innovadores (como la asociación para la innovación) o la ejecución de los proyectos. En segundo término, difunde buenas prácticas e información sobre la cuestión. En este ámbito destaca la Toolbox, herramienta electrónica creada en cooperación con la Universidad del Ejército Alemán de Múnich que ofrece aplicaciones sobre aspectos como el diseño de una estrategia de adquisiciones innovadora o el cálculo de costes del ciclo de vida de las adquisiciones. En tercer término, organiza eventos en todo el país. Estos se dividen en Innovationsschauplätze centrados en presentar la compra pública innovadora en las distintas administraciones que lo requieran y Strategische Dialoge, en los que representantes y expertos de organizaciones del sector público y privado intercambian experiencias. Además, participan en distintas ferias regionales. Por último, ofrece formación a través de se-

12 «La denominada "profesionalización" solicitada por la Comisión, es decir, la formación del personal de las administraciones, recae exclusivamente en la soberanía organizativa de los Estados miembros y, en particular, de los entes locales y regionales; además, expresa su temor de que una mayor cantidad de directrices y manuales de la Comisión sobre la profesionalización de la contratación pública (como, por ejemplo, el amplio marco europeo de profesionalización previsto actualmente) implicaría una mayor carga administrativa para los poderes adjudicadores, que se sumaría a la del ya de por sí extenso marco normativo» (Comité Europeo de las Regiones, 2020).

13 Das zentrale Portal für nachhaltige Beschaffung öffentlicher Auftraggeber. http://www.nachhaltige-beschaffung.info/DE/Home/ home_node.html

$\overline{14}$ Das Kompetenzzentrum innovative Beschaffung - KOINNO. https://www.koinno-bmwi.de 
minarios cortos, centrados en cuestiones sobre adquisiciones estratégicas o procedimientos negociados y cursos de capacitación específica mediante e-learning.

Extender la formación y el intercambio de experiencias más allá de los límites de la Administración pública es deseable de cara a asegurar una contratación plenamente respetuosa con el principio de integridad (OCDE, 2015, p. 7), además de resultar fundamental para la consecución de algunos objetivos vinculados a la contratación pública estratégica, como la participación de las pequeñas y medianas empresas (Saastamoinen et al., 2017). En este sentido, cabe destacar la experiencia impulsada por el Forum Vergabe, organización sin ánimo de lucro fundada en Alemania en 1993 que, valiéndose únicamente de las cuotas de sus socios, mantiene una plataforma dedicada al intercambio de experiencias y buenas prácticas entre los profesionales dedicados a la contratación, desde la perspectiva tanto de licitadores como de contratistas a partir de grupos de discusión nacionales y regionales. Además de servir para el intercambio de conocimientos, el Forum publica estudios, realiza informes a solicitud de sus miembros y organiza cursos y eventos. La institución concede un prestigioso premio de contratación pública internacional destinado a jóvenes académicos con una dotación de 5.000 euros.

\subsection{El suministro de herramientas y metodologías adecuadas}

El tercero de los tres ejes en los que se basa la recomendación hace referencia a la necesidad de suministrar herramientas, mencionando cuatro aspectos concretos:

En primer término, el estímulo y apoyo al desarrollo y la aceptación de herramientas tecnológicas accesibles para simplificar y mejorar el funcionamiento de los sistemas de contratación a través, por ejemplo, de la creación de portales de internet únicos para permitir el acceso a la información o de herramientas específicas para fomentar economías de escala, eficiencia energética, trabajo en equipo u otras soluciones orientadas al mercado. También, dentro de este ámbito, menciona el impulso a un planteamiento estratégico para la digitalización mediante la normalización, el intercambio, la reutilización y la interoperabilidad de productos y servicios.

En segundo término, el apoyo y la promoción de la integridad mediante mecanismos como el establecimiento de códigos deontológicos y cartas para la integridad, la utilización de datos sobre irregularidades y la retroalimentación para desarrollar programas de formación y orientaciones. Asimismo, promueve el self-cleaning y el desarrollo de orientaciones para prevenir y detectar supuestos de fraude y corrupción, en conexión con el establecimiento de canales de denuncia.

En tercer término, el apoyo a la orientación por parte de los Estados en dos sentidos. En primer lugar, con el fin de promover la seguridad jurídica sobre legislación y requisitos legales a nivel estatal y europeo respecto a obligaciones internacionales. En segundo lugar, con el de facilitar y fomentar el pensamiento estratégico, el criterio comercial y la toma de decisiones inteligentes. Ello mediante la elaboración de material de orientación específico, manuales de metodología, depósitos de buenas prácticas y errores más comunes actualizados, fácilmente utilizables, accesibles y basados en la experiencia profesional, plantillas normalizadas y herramientas para procedimientos como criterios de contratación publica ecológica o socialmente responsable.

En cuarto término, el fomento del intercambio de buenas prácticas y el apoyo a los profesionales para garantizar procedimientos de contratación profesionales, trabajo cooperativo y transmisión de conocimientos técnicos. Ello a través del establecimiento de servicios de ayuda técnica reactivos, la organización de seminarios y talleres y la creación de foros de internet y redes sociales profesionales.

Respecto a este eje cabe destacar la experiencia del Expertisecentrum Aanbesteden o Centro de Expertos en Adquisiciones del Ministerio de Asuntos Económicos y Clima de los Países Bajos (PIANOo). En 1995 se constituyó como una red de aproximadamente 3.500 poderes adjudicadores que sirve como fuente de información y experiencias. Su página electrónica, en la que podemos encontrar distintas herramientas para fomentar el intercambio de buenas prácticas e información entre licitadores (como el PIANOo Forum), sirve de base para sus actividades. PIANOo impulsa tareas muy diversas: desde la organización de un congreso anual y varias reuniones periódicas enfocadas en temas de interés hasta la formación, mediante la celebración anual del Leergang Aanbestedingsrecht o curso de Derecho de la contratación pública, además de la divulgación, a través de diversas publicaciones (PIANOo, Expertisecentrum Aanbesteden, s. f.).

La necesidad de que existan instituciones dedicadas a integrar y coordinar políticas públicas en materia de contratación con independencia del nivel de descentralización que exista en un determinado Estado, ha sido puesta de manifiesto por la doctrina (Kauppi y Van Raaij, 2015, p. 953). Entre otros aspectos puede con- 
tribuir de forma decisiva a preservar la integridad en el sistema de contratación, sirviendo de «cortafuegos que evita el contacto directo entre suministradores y usuarios» (OCDE, 2009a, p. 29). En este sentido cabe destacar la experiencia desarrollada en Nueva Zelanda (primer país del mundo en eficacia en materia de contratación pública según el InCiSE 2019) a partir de la puesta en marcha del New Zealand Procurement Capability Index $(\mathrm{PCl})^{15}$.

Se trata de una herramienta diseñada para evaluar y reforzar la capacidad para realizar compras públicas por las distintas agencias de la administración neozelandesa. Estas se autoevalúan periódicamente, lo que permite a quienes las dirigen tener una visión transversal de las debilidades y fortalezas con las que cuentan identificando las distintas oportunidades de mejora y supervisar su evolución, así como incrementar el liderazgo de los gerentes (OCDE, 2015). En el proceso participan la práctica totalidad de los empleados que trabajan en la contratación, tomando en consideración distintos parámetros que cubren el ciclo de adquisición. Concretamente se evalúan: estrategia y resultados, resultados comerciales y sociales, gobernanza, riesgos y beneficios, planificación y abastecimiento, gestión de resultados, gestión de recursos humanos y capacidad de reestructuración. Los resultados de la autoevaluación se atenúan y modulan a partir de la revisión externa que lleva a cabo el New Zealand Goverment Procurement (NZGP), organismo gubernamental especializado en contratación pública.

Los datos se centralizan -lo que implica que puede obtenerse en todo momento una visión global y transversal de la contratación pública en Nueva Zelanda- y a partir de su análisis se identifican las áreas que requieran realizar cambios e impulsar mejoras en cada Agencia. En base a ello, el NZGP ofrece programas formativos adaptados a las debilidades detectadas, además de poner en contacto a las que alcanzan un mayor nivel de eficiencia con las que presentan problemas. Aunque no todas las agencias están obligadas a remitir sus datos (por ejemplo, en el caso de que tengan un bajo índice de actividad en materia de contratación) se anima a todas a participar en el proceso, por lo que existe una herramienta de autoevaluación diseñada para organizaciones de pequeño tamaño. El PCl supone un $5 \%$ de la puntuación total que las distintas agencias pueden obtener dentro del Investor Confidence Rating assesment (ICR), una evaluación trienal que el Tesoro público realiza estimando la capacidad que tienen las agencias para gestionar sus inversiones y activos.

\section{CONCLUSIONES}

La profesionalización es una tarea heterogénea, compleja e imprescindible para garantizar el éxito de la contratación pública estratégica, así como su eficacia en situaciones de emergencia. También es una exigencia legal contenida en el artículo 334.1.e) LCSP. Dada su complejidad y el contenido de la Recomendación de la Unión Europea de 2017 sobre la cuestión deben tenerse en cuenta en el diseño futuro de la ENCP las experiencias de éxito que han tenido lugar en otros países.

La creación de un cuerpo de gerentes especializados en prestar labores de asistencia a las distintas Administraciones en la implementación y desarrollo de los procedimientos de licitación pública al estilo de la Government Commercial Function británica, podría mejorar considerablemente la orientación estratégica de la contratación en las distintas administraciones, con independencia de su capacidad económica y técnica para invertir en la profesionalización (acusadamente menor en el ámbito de las entidades locales).

Es preciso innovar en los procedimientos de selección de profesionales dedicados a la contratación. En primer lugar, normalizando y facilitando la captación del talento al tiempo que se minimiza el coste personal y económico que para un joven graduado puede tener el clásico procedimiento de la oposición. Plataformas como la Talent Cloud canadiense o la SELOR belga son un ejemplo en este sentido. En segundo lugar, abandonando pruebas estrictamente memorísticas para incentivar el acceso de los más capacitados incluso a partir de procesos de preselección realizados en la universidad complementados con la realización de prácticas estivales, como en el caso del Fast Stream británico.

Diferentes estudios revelan la insuficiencia de formación específica. Respecto a la inicial destacan las experiencias australiana, canadiense o estadounidense, en las que las Escuelas de administración pública diseñan programas divididos en distintos niveles a superar como parte de la formación inicial antes de la asignación de un puesto de trabajo. Respecto a la continua destaca la experiencia alemana a través de del KNB o el KOINNO. Además de ofrecer una formación continua especializada para los profesionales de la

15 Delivering Better Outcomes for New Zealand. https://www.procurement.govt.nz 
contratación con independencia de donde desarrollen su actividad, estas instituciones públicas especializadas permiten a las distintas Administraciones diseñar políticas de contratación pública estratégica adaptadas a sus objetivos prioritarios.

Por último, cabe destacar la necesidad de mejorar la gestión de las organizaciones y sus recursos humanos para garantizar el éxito de la profesionalización, lo que requiere una monitorización y análisis de la información -como ocurre en Escocia mediante el PCF-que sirvan de base a una continua evaluación de la actividad, impulsada en Nueva Zelanda a través del PCI. Ello no puede desligarse de una evaluación del desempeño adaptada a la particular idiosincrasia de la contratación pública y ligada tanto al desarrollo de la carrera profesional como al diseño de programas de formación específicos, que sirva para hacer frente a las debilidades detectadas.

\section{REFERENCIAS BIBLIOGRÁFICAS}

Bandiera, O., Prat, A. y Valletti, T. M. (2009). Active and passive waste in government spending: evidence form a policy experiment. American Economic Review, 99(4),1.278-1.308. https://doi.org/10.2139/ssrn.1115339. https://doi. org/10.1257/aer.99.4.1278

Blavatnik School of Government y The Institute for Goverment (2020). The international civil service effectiveness (InCiSE) Index [Results Report 2019]. Universidad de Oxford. https://www.bsg.ox.ac.uk/sites/default/files/2019-04/ InCiSE\%202019\%20Results\%20Report.pdf

Cantero Martínez, M. J. (2020). La profesionalización de la contratación pública como herramienta de innovación. En I. Martín Delgado y J. A. Moreno Molina (dirs.), Administración electrónica, transparencia y contratación pública. lustel.

Cerrillo i Martínez, A. (2014). El principio de integridad en la contratación pública. Mecanismos para la prevención de los conflictos de intereses y la lucha contra la corrupción. Aranzadi.

Charrón, N., Dahlström, C., Fazekas, M. y Lapuente, V. (2017). Careers, Connections, and Corruption Risks: Investigating the Impact of Bureaucratic Meritocracy on Public Procurement Processes. The Journal of Politics, 79(1) 89-104, https://doi.org/10.1086/687209

Christensen, B. A. (1994). What is a profession? Journal of the American Society of CLU \& ChFC, 48(1), 28.

Comité Europeo de las Regiones, Comisión de Política Económica (5 de febrero de 2020). Dictamen del Comité Europeo de las Regiones - Informe sobre la aplicación de la contratación pública (2020/C 39/09). Diario Oficial de la Unión Europea. Recuperado el 31 de julio de 2020 de https://eur-lex.europa.eu/legal-content/ES/TXT/?uri=urise rv:OJ.C_.2020.039.01.0043.01.SPA

Comisión Europea (CE) (2016). Stock-taking of administrative capacity, systems and practices across the EU to ensure the compliance and quality of public procurement involving European Structural and Investment (ESI) Funds. Publicaciones Oficiales de la Unión Europea. Recuperado el 31 de julio de 2020 de https://op.europa.eu/en/ publication-detail/-/publication/d1082259-0202-11e6-b713-01aa75ed71a1

Cooper, C. A. (2008). Encouraging civil servants to be frank and fearless: Merit recruitment and employee voice. Public Administration 96(4), 721-735 https://doi.org/10.1111/padm.12548

Coviello, D. y Mariniello, M. (2014). Publicity requirements in public procurement: evidence from a regression discontinuity desing. Journal of Public Economics, 109, 76-100, https://doi.org/10.1016/j.jpubeco.2013.10.008

Diario Oficial de la Unión Europea (28 del 6 de 2018). Dictamen del Comité Económico y Social Europeo sobre la recomendación de la Comisión, de 3 de octubre de 2017, sobre la profesionalización de la contratación pública Construir una arquitectura para la profesionalización de la contratación pública [C(2017) 6654 final-SWD(2017), 327 final]. Recuperado el 31 de julio de 2020 de https://eur-lex.europa.eu/legal-content/ES/TXT/PDF/?uri=CELEX \%3A52017AE5294\&from=ES

Eddy, S. W. NG. y Gossettm, CH. W. (2013). Career Choice In Canadian Public Service: An Exploration Of Fit With The Millennial Generation. Public Personnel Management, 42(3), 337-358. https://doi.org/10.1177/0091026013495767

Evans, P. y Rauch, J. E. (1999). Bureaucracy and Growth: A Cross-National Analysis of the Effects of «Weberian» State Structures on Economic Growth. American Sociological Review, 64(5), 748-765, https://doi.org/10.2307/2657374

Federal Acquisiton Institute (2019). Contracting Officer's Representative (FAC-COR). Recuperado el 31 de julio de 2020 de https://www.fai.gov/certification/fac-cor

García Jiménez, A. (2018). Las conexiones entre contratación administrativa e innovación. Tecnos.

Gimeno Feliu, J. M. (2016). El nuevo paquete legislativo comunitario de contratación pública: principales novedades (la orientación estratégica de la contratación pública). En S. Rodríguez-Camos (coord.), Las nuevas directivas de contratos públicos y su transposición (pp. 15-127). Marcial Pons.

Glas, A. H., Schaupp, M. y Essig, M. (2017). An organizational perspective on the implementation of strategic goals in public procurement. Journal of public procurement, 17(4), 572-605. https://doi.org/10.1108/jopp-17-04-2017-b004

Gobierno de Canadá (s. f.). Directive on Mandatory Training. Recuperado el 31 de julio de 2020 de https://www.tbs-sct. gc.ca/pol/doc-eng.aspx?id=32628 
Jiménez Asensio, J. M. (2018). «Repensar» la selección de empleados públicos: momento actual y retos de futuro. Pertsonak eta Antolakunde Publikoak kudeatzeko Euskal Aldizkaria (Revista Vasca de Gestión de Personas y Organizaciones Públicas), número especial 2, 8-29. https://apps.euskadi.eus/t59auUdaWar/R3/verArticulo?nume jem $=14 \&$ tipo $=R \&$ seccion $=51 \&$ correlativo $=3 \&$ contenido $=12 \&$ locale $=e s$

Kauppi, K. y Van Raaij, E. M. (2015). Opportunism and Honest Incompetence - Seeking Explanations for Noncompliance in Public Procurement. Journal of Public Administration Research and Theory, 25(3), 953-979. https://doi.org/10.1093/jopart/mut081

Kline, E. (1981). To be a professional. Southern Review of Public Administration, 5(3), 258-281. https://www.jstor.org/ stable/40860033

Krause, E. A. (1996). Death of the Guilds: Professions, States and the Advance of Capitalism, 1930 to the Present. Yale University Press. http://www.jstor.org/stable/j.ctt1dszxkq

Malaret i García, E. (2016). El nuevo reto de la contratación pública para afianzar la integridad y el control: reforzar el profesionalismo y la transparencia. Revista Digital de Derecho Administrativo, 15, 21-60. https://doi. org/10.18601/21452946.n15.04

Mapelli Marchena, C. (2018). La visión comparada: Nuevos sistemas de selección de la alta función pública en las democracias avanzadas. Pertsonak eta Antolakunde Publikoak kudeatzeko Euskal Aldizkaria (Revista Vasca de Gestión de Personas y Organizaciones Públicas), número especial 2, 32-50. https://apps.euskadi.eus/ t59auUdaWar/R3/verArticulo?numejem=14\&tipo=R\&seccion=51\&correlativo=2\&contenido=6\&locale=es

Oficina Independiente de Regulación y Supervisión de la Contratación - OIRESCON) (2020). Informe anual de supervisión de la contratación pública de España. Diciembre 2020. https://www.hacienda.gob.es/RSC/OIReScon/ informe-anual-supervision-2020/ias-2020.pdf

Organización para la Cooperación y el Desarrollo Económicos (OCDE) (2009a). OECD Principles for Integrity in Public Procurement. https://www.oecd.org/gov/ethics/48994520.pdf

Organización para la Cooperación y el Desarrollo Económicos (OCDE) (2009b). La integridad en la contratación pública: buenas prácticas de la A a la Z. https://www.oecd.org/fr/gov/ethique/38947794.pdf

Organización para la Cooperación y el Desarrollo Económicos (OCDE) (2015). Recommendation of the council of public procurement. https://www.oecd.org/gov/public-procurement/OECD-Recommendation-on-Public-Procurement.pdf

Organización para la Cooperación y el Desarrollo Económicos (OCDE) (2019a). Public Procurement in Germany. Strategic Dimensions for Well-Being and Growth (OECD Public Governance Reviews). OECD Publishing. https:// doi.org/10.1787/1db30826-en

Organización para la Cooperación y el Desarrollo Económicos (OCDE) (2019b). Reforming Public Procurement. Progress in implementing the 2015 OECD Recommendation (OECD Public Governance Reviews). OECD Publishing. https://doi.org/10.1787/1de41738-en

Pianoo, Expertisecentrum Aanbesteden (s. f.). Ministerie van Economische Zaken en Klimaat. Recuperado el 31 de julio de 2020 de https://www.pianoo.nl/nl

Rastrollo Suárez, J. J. (2018). Evaluación del desempeño en la Administración: hacia un cambio de paradigma en el sistema español de empleo público. Tirant lo blanch.

Rivero Ortega, R. (2012). La necesaria innovación en las instituciones administrativas. INAP.

Sanmartín Moa, M. A. (2012). La profesionalización de la contratación pública en el ámbito de la Unión Europea. En M. A. Bernal Blay (coord.) y J. M. Gimeno Feliú (dir.). Observatorio de contratos públicos 2011 (pp. 407-429). Civitas.

Saastamoinen, J., Reijonen, H. y Tammi, T. (2017). The role of training in dismantling barriers to sme participation in public procurement. Journal Of Public Procurement, 17(1), 1-30. https://doi.org/10.1108/JOPP-17-01-2017-B001

Steinfeld, J. M., Prier, E. y McCue, C. (2015). Public procurement and the BLS: operationalizing occupational duties. International Journal Of Public Sector Management, 28(7), 510-527. https://doi.org/10.1108/IJPSM-12-2014-0150

Steinfeld, J. M., Prier, E. y McCue, C. (2016). Examining professionalization in public procurement by ranking practitioner job positions according to job activities. International Journal of Procurement Management, 9(3), 328353, https://doi.org/10.1504/IJPM.2016.076308

TechFAR Hub (2020). Digital IT Acquisition Professional Training [Initiative]. Recuperado el 31 de julio de 2020 de https://techfarhub.cio.gov/initiatives/ditap/

Treasury Board of Canada Secretariat (6 de diciembre de 2019). Evaluation of the Certification Program for Procurement and Materiel Management Communities in the Government of Canada [Report]. Recuperado el 31 de julio de 2020 de https://www.canada.ca/en/treasury-board-secretariat/corporate/reports/evaluation-certificationprogram-procurement-materiel-management-communities-2019.html

Wayan Rustiarini, N., Sutrisno, T., Nurkholis, N. y Andayani, W. (2019). Why people commit public procurement fraud? The fraud diamond view. Journal of public procurement, 19(4), 345-362. https://doi.org/10.1108/JOPP-02-2019-0012

Zussman, D. (2002). Alternative Service Delivery. En C. Dunn (ed.), The Handbook of Canadian Public Administration (pp. 53-76), Oxford University Press. 